\title{
Improving osteoporosis assessment in the fracture
} clinic

\section{CP CHARALAMBOUS, C MOSEY, E JOHNSTONE, P AKIMAU, TK GULLETT, I SIDDIQUE, RA WILKES}

\author{
Department of Trauma and Orthopaedics, Hope Hospital, University of Manchester, Salford, UK
}

ABSTRACT

INTRODUCTION The aim of this study was to compare the effectiveness of different ways of referring patients to an osteoporosis assessment service at an orthopaedic fracture clinic of a hospital in the UK.

PATIENTS AND METHODS Three methods of identifying and referring to an osteoporosis assessment service were evaluated. RESULTS Relying on doctors for such a referral gave a catchment rate of only $1.6 \%$. Involving patients themselves, asking them to self-refer, increased the catchment rate to $63 \%(P<0.0001)$. Having a specialist osteoporosis and fracture liaison nurse present in clinic and reviewing the notes of patients checking in, to see if they match criteria for osteoporosis assessment, further increased catchment to $77 \%(P=0.036)$.

CONCLUSIONS Simply having an osteoporosis assessment service and strict criteria to identify which patients should be referred to such a service will not necessarily increase catchment rate for osteoporosis patients. A nurse physically present in the clinic provided the best result, and supports the need of investing in an osteoporosis and fracture liaison nurse.

\section{KEYWORDS}

Osteoporosis - Fracture clinic - Assessment

\section{CORRESPONDENCE TO}

Pavel Akimau, Department of Trauma and Orthopaedics, Hope Hospital, University of Manchester, Stott Lane, Salford M6 8HD, UK E: pavel.akimau@doctors.org.uk

Osteoporosis is a major cause of fractures. Patients sustaining one osteoporosis related fracture have an increased risk of further fractures of both the axial and appendicular skeleton. Prophylactic treatment in such patients significantly reduces the risk of re-fracturing. ${ }^{1-4}$

Patients presenting to hospitals in the UK with a fracture are usually only seen by the orthopaedic team without any input from medical physicians. Assessment for osteoporosis should, in principle, be an integral part of any orthopaedic fracture service. Previous studies have shown that the rate of appropriate investigation and management of patients with osteoporosis in the orthopaedic department is very low and that the majority of individuals who sustain fragility fractures are not receiving adequate osteoporosis management. ${ }^{5-8}$ Several hospital trusts in the UK have put in place osteoporosis assessment services, often run by a specialist nurse, aiming at further investigating (with DEXA scan) and, where necessary, commencing osteoporosis treatment for patients presenting with fractures. However, a major challenge is identifying those patients who might benefit and referring them to such the osteoporosis assessment service.

The aim of this study was to compare the effectiveness of different ways of referring patients to an osteoporosis assessment service for patients presenting with possible osteoporosis-related fractures at the orthopaedic fracture clinic of a hospital in the UK.

\section{Patients and Methods}

At Hope Hospital, Salford, UK, an osteoporosis and fracture liaison specialist nurse provides an osteoporosis assessment and triage service which involves risk assessment, referral for a DEXA scan and, where necessary, commencement of treatment in collaboration with the medical team and the patient's general practitioner. According to our protocol, any patient over the age of 50 years presenting to the fracture clinic with a fracture should be referred to this osteoporosis assessment service. It is worth noting that, in our hospital, many patients presenting to the fracture clinic may not have a fracture but are elective follow-up patients. We looked at three different ways in which patients presenting to our fracture clinic who were older than 50 years and had a fracture could be identified and referred to this osteoporosis assessment service. Each arm of the study was applied for 1 week, after a trial period.

\section{Arm 1}

Doctors attending fracture clinics were asked to refer to the 
Table 1 Proportion of patients meeting referral criteria (older than 50 years and presenting with a fracture) sent to the osteoporosis assessment service

Trial arm 1

doctors referring

$1 / 59(1.6 \%)$
Trial arm 2

patients self-referring + doctors referring

$28 / 44(63 \%)^{a}$
Trial arm 3

osteoporosis and fracture liaison

nurse present in clinic.

$39 / 51(77 \%)^{b}$

${ }^{\mathrm{a}} P=<0.0001$, trial arm 2 versus $1 ;{ }^{\mathrm{b}} P=0.036$, trial arm 3 versus 2.

osteoporosis and fracture liaison nurse any patient over the age of 50 years presenting to the clinic with a fracture using a pre-designed proforma. Doctors attending fracture clinics were given group and one-to-one tutorials as to the criteria and method for referral.

\section{Arm 2}

In addition to doctors referring, all patients checking into the fracture clinic were given a leaflet explaining what osteoporosis is and why it might affect them if they had sustained a fracture. They were asked to self-register for osteoporosis assessment if they were older than 50 years and attending the clinic because of a fracture, using a predesigned proforma.

\section{Arm 3}

The osteoporosis and fracture liaison specialist nurse was present in clinic and assessed the medical records of each patient checking in, to see if the patient was older than 50 years and had a fracture. If this was the case, the patient was, at the same clinic visit, asked to undergo osteoporosis assessment, which the osteoporosis nurse herself went on to perform.

After completion of the three arms of the study, review of the clinic letters identified the number of patients that should have been referred to the osteoporosis assessment service. Statistical analysis was performed using binomial test, with SPSS (v.16.0; SPSS Inc.) software The significance level was established at a $P$-value of less that $0.05 \%$.

\section{Results}

As shown in Table 1, relying on doctors to refer patients to the osteoporosis assessment service produced a very low catchment rate of only $1.6 \%$. Involving patients themselves increased this significantly to $63 \%(P<0.0001)$. Having the osteoporosis nurse physically present in clinic going through each patient's records further increased catchment to $77 \%(P=0.036)$.

\section{Discussion}

Our results accord with previous studies reporting that relying on doctors to identify at-risk patients gives a very poor yield. It was interesting to show that involving patients themselves significantly increased catchment. However, having a person (in our case an osteoporosis and fracture liaison specialist nurse) dedicated to examining each patient's notes upon arrival to the clinic gave the highest catchment rate. The osteoporosis assessment consultation was provided by the same specialist nurse, which meant that it could be performed on the same day rather than relying on telephone or mail contact at a later date. It was of note that, despite the osteoporosis and fracture liaison nurse being present in clinic, we achieved only $77 \%$ rather than $100 \%$ catchment rate. This was mainly due to the workload that the specialist nurse had to face, with many patients checking in at the same time, or patients checking in while the osteoporosis nurse was consulting with a patient. Increasing the resources and having a second person to act as osteoporosis nurse assistant could potentially further increase catchment.

Previous studies have reported on automatic referral of patients attending fracture clinics using standardised referral letters. ${ }^{9}$ Nevertheless, in a setting such as ours where many patients presenting to the fracture clinic may not have a fracture, a system of identifying patients meeting the referral criteria is still necessary and resource-consuming. We also feel that a one-stop visit where osteoporosis management is done alongside fracture management may increase patients' compliance to osteoporosis interventions.

\section{Conclusions}

This study suggests that simply having an osteoporosis assessment service and strict criteria to identify which patients should be referred to such a service will not necessarily increase catchment rate for osteoporosis patients. Our study has confirmed that relying on doctors to refer such patients to the osteoporosis assessment service gives a 
poor yield. Involving the patients themselves increases catchment rate. Nevertheless, having an osteoporosis nurse physically present in the clinic provided the highest yield, and supports the need of investing in an osteoporosis and fracture liaison nurse. ${ }^{10}$ Our study emphasises that individual needs for each hospital must be examined, and any osteoporosis protocol must be geared to those needs.

\section{References}

1. Black DM, Thompson DE, Bauer DC, Ensrud K, Musliner T, Hochberg MC et al. Fracture risk reduction with alendronate in women with osteoporosis: the Fracture Intervention Trial. FIT Research Group. J Clin Endocrinol Metab 2000; 85: 4118-24.

2. Chesnut III CH, Skag A, Christiansen C, Recker R, Stakkestad JA, Hoiseth A et al.; Oral Ibandronate Osteoporosis Vertebral Fracture Trial in North America and Europe (BONE). Effects of oral ibandronate administered daily or intermittently on fracture risk in postmenopausal osteoporosis. J Bone Miner Res 2004; 19: 1241-9.

3. Hochberg MC, Thompson DE, Black DM, Quandt SA, Cauley J, Geusens P et al.; FIT Research Group. Effect of alendronate on the age-specific incidence of symptomatic osteoporotic fractures. J Bone Miner Res 2005; 20: 971-6.

4. Tang BM, Eslick GD, Nowson C, Smith C, Bensoussan A. Use of calcium or calcium in combination with vitamin $\mathrm{D}$ supplementation to prevent fractures and bone loss in people aged 50 years and older: a meta-analysis. Lancet 2007; 370: 657-66.

5. Ashe MC, Janssen P, Guy P, Khan KM, Improving osteoporosis management in at-risk fracture clinic patients. J Am Geriatr Soc 2005; 53: 727-8.

6. Freedman BA, Potter BK, Nesti LJ, Cho T, Kuklo TR. Missed opportunities in patients with osteoporosis and distal radius fractures. Clin Orthop 2007; 454: 202-6.

7. Giangregorio L, Papaioannou A, Cranney A, Zytaruk N, Adachi JD. Fragility fractures and the osteoporosis care gap: an international phenomenon. Semin Arthritis Rheum 2006; 35: 293-305.

8. Talbot JC, Elener C, Praveen P, Shaw DL. Secondary prevention of osteoporosis: calcium, vitamin $\mathrm{D}$ and bisphosphonate prescribing following distal radial fracture. Injury 2007; 38: 1236-40.

9. Skedros JG. The orthopaedic surgeon's role in diagnosing and treating patients with osteoporotic fractures: standing discharge orders may be the solution for timely medical care. Osteoporos Int 2004; 15: 405-10.

10. Larsson S. Time to invest in a 'fracture liaison nurse'. Injury 2007; 38 : $1225-6$.

\section{New online only case reports}

You can access the case reports by using your College-issued Athens username and password to enter the members' area of the College website (<www.rcseng.ac.uk/members/annals/>) and following the link to the Annals.

Alternatively, if you type the following URL into the address bar of your web browser $<\mathrm{http}: / / \mathrm{dx}$.doi.org/> and then enter the DOI in the dialogue box presented on this web page, you will be taken directly to the abstract of the article.

Case report: Gamma probe radioguided parathyroid forearm surgery in recurrent hyperparathyroidism RI CUTRESS, C MANWARING-WHITE, K DIXON, A DHIR, AI SKENE doi 10.1308/147870809X401047

Case report: Slipped upper femoral epiphysis - a case of a 'dodgem hip' DUSHAN THAVARAJAH, RAJA SEKHAR MAROJU, LOKESH NARAYANASWAMY doi 10.1308/147870809X450593

Case report: Superior mesenteric artery syndrome in association with an abdominal aortic aneurysm A PAL, AEP CAMERON

doi 10.1308/147870809X450575

Case report: Squamous cell carcinoma presenting as peri-implantitis: a case report AAKSHAY GULATI, FRANCY J PUTHUSSERY, IAN P DOWNIE, TIMOTHY R FLOOD 